\title{
The chiral condensate of strongly coupled QCD in the 't Hooft limit
}

\author{
G.Grignani, D.Marmottini, and P.Sodano \\ Dipartimento di Fisica and Sezione I.N.F.N. Universitá di Perugia, \\ Via A. Pascoli,06123 Perugia, Italy
}

(Dated: November 6, 2018)

\begin{abstract}
Using the recently proposed generalization to an arbitrary number of colors of the strong coupling approach to lattice gauge theories [1], we compute the chiral condensate of massless QCD in the 't Hooft limit.

PACS numbers: 11.10.Ef, 11.15.Me, 11.15.Pg, 12.38.Gc, 14.40.Cs

Keywords: lattice, chiral condensate, large N.
\end{abstract}




\section{INTRODUCTION}

As it is well known, the spontaneous breakdown of the chiral symmetry in massless QCD is signaled by the appearance of a non-vanishing order parameter, the chiral condensate $\chi_{L}=<\bar{\Psi} \Psi>$. This quantity is intrinsically non perturbative and its determination is not at all straightforward. Since numerical computations on the lattice yield -at a given fixed lattice spacing- only a bare result, one needs renormalization in order to use the result for phenomenological applications. The results of several recent studies [2, 3, 4, 5] evidence by now the dependence of the chiral condensate on both the scale and the renormalization scheme but allow for the computation of a scale independent Renormalization Group Invariant (RGI) chiral condensate [3]. Recently, an interesting analytical evaluation of the quark condensate in one-flavor massless QCD from the value of the gluino condensate in SUSY Yang-Mills theory by means of orientifold large- $N$ expansion has appeared in the literature [6].

In this paper we compute the bare chiral condensate in the Hamiltonian formulation of lattice QCD using staggered fermions in the strong coupling limit of the theory for any value of $N_{C}$; the lattice chiral condensate is evaluated using $1 / g^{2}$ as the expansion parameter and, then, taking the 't Hooft limit (large $N_{C}$, with $g^{2} N_{C}$ fixed). The extrapolation to the continuum limit is carried out by means of Padé approximants. The tools used for our analysis were developed in [1]; here we shall present only the result for the chiral condensate referring the reader to [1] for the details of the method.

The result obtained for the chiral condensate $\chi_{L}$ is compared to the recent numerical determination of the RGI chiral condensate [3]. In particular, we estimate the scale independent and dimensionless ratio between $\chi_{L}$ and the cube of the mass of the $\rho$ meson, which has been obtained in a strong coupling large $N_{C}$ calculation in 1], and compare it to the numerical value of the ratio between the scale independent RGI chiral condensate obtained in [3] and the experimental value of the cube of the mass of the $\rho$ meson; the check allows for an estimate of the lattice light velocity $t$ on the lattice. $t$ turns out to be equal to 1.025 , in excellent agreement with the expected result of 1 . This result in turn confirms the accuracy of our previous evaluation of the mass spectrum of mesons [1].

The strong coupling limit, where the hadrons are automatically confined, is a natural starting point for the study of the long-distance, non-perturbative features of the chiral 
symmetry breaking in massless QCD. Even if, at weak coupling, the Wilson formalism yields an accurate analysis of the $U(1)$ anomaly and PCAC 7], one cannot use it to compute the chiral condensate at strong coupling since it explicitly breaks the chiral symmetry on the lattice.

The staggered fermion formalism yields, instead, a remnant of chiral symmetry on the lattice, which is the invariance of the theory under translation by a single link; thus, a spontaneous breakdown of chiral symmetry may be evidenced also at strong coupling via a non vanishing chiral condensate. Moreover, the staggered fermion formalism is known to yield good results in the strong coupling evaluation of the hadron spectrum [1, 8, 9] and of chiral condensate of lower dimensional models [10, 11] Other types of lattice fermions such as domain-wall or overlap fermions are expected to suffer both doubling and explicit breaking of chiral symmetry[12] at strong coupling.

Strongly coupled lattice gauge theories are intimately related to quantum spin systems [13]; in particular lattice gauge theories with staggered fermions exhibit interesting similarities with condensed matter systems. For example, it is well known that the quantum spin-1/2 Heisenberg antiferromagnet is equivalent to the strong coupling limit of either a $U(1)$ or $S U(2)$ lattice gauge theory [14, 15, 16]. For the gauge group $U\left(\mathcal{N}_{c}\right)$, one may establish the equivalence with a spin- $\mathcal{N}_{C} / 2$ Heisenberg antiferromagnet [17]. Since the seminal work of 't Hooft [18, 19] the large $N_{C}$ limit (with $g^{2} N_{C}$ fixed), has played an increasingly important role in studying gauge theories in the continuum, on the lattice [1, 20] and the duality between gauge and string theories [21, 22]. Furthermore, in a recent paper 1], we have generalized the strong coupling calculation by Banks et al. [8] to an arbitrary number of colors $N_{C}$ and we have used the 't Hooft limit to investigate some features of the meson spectrum of strongly coupled lattice QCD. Our results imply that, also at strong coupling, the 't Hooft limit offers a very accurate method to consistently determine both the QCD spectrum and the chiral condensate.

\section{STRONG COUPLING EVALUATION OF THE QCD CHIRAL CONDENSATE IN THE 'T HOOFT LIMIT}

In the Hamiltonian formulation of lattice QCD with staggered fermions [23] time is a continuous variable and space is discretized on a 3-dimensional cubic lattice with $M$ sites, 
labeled by $\vec{r}=(x, y, z)$; with $x, y$ and $z$ integers. The lattice Hamiltonian with one flavor of massless quark may be written as the sum of three contributions

$$
H=H_{e}+\tilde{H}_{q}+H_{m}
$$

where

$$
\begin{aligned}
& H_{e}=\frac{g^{2}}{2 a} \sum_{[\vec{r}, \hat{n}]} E^{a}[\vec{r}, \hat{n}]^{2} \\
& \tilde{H}_{q}=\frac{t}{2 a} \sum_{[\vec{r}, \hat{n}]} \eta(\hat{n}) \Psi_{A}^{\dagger}(\vec{r}+\hat{n}) U_{A B}[\vec{r}, \hat{n}] \Psi_{B}(\vec{r})+\text { h.c. } \equiv H_{q}+H_{q}^{\dagger} \\
& H_{m}=\frac{1}{2 g^{2} a} \sum_{[\vec{r}, \hat{n}, \hat{m}]}\left[\operatorname{Tr}\left(U[\vec{r}, \hat{n}] U[\vec{r}+\hat{n}, \hat{m}] U^{\dagger}[\vec{r}+\hat{m}, \hat{n}] U^{\dagger}[\vec{r}, \hat{m}]\right)+\text { h.c. }\right]
\end{aligned}
$$

are the electric field Hamiltonian, the interaction Hamiltonian between quarks and gauge fields and the magnetic Hamiltonian, respectively. $t$ is the lattice light velocity. The sums $\sum_{[\vec{r}, \hat{n}]}$ are extended to the $N$ lattice links, whereas $\sum_{[\vec{r}, \hat{n}, \hat{w}]}$ is a sum over the plaquettes. $\hat{n}=\hat{x}, \hat{y}, \hat{z}$ is the unit vector in the $\vec{n}$ direction and

$$
\eta(\hat{x})=(-1)^{z}, \quad \eta(\hat{y})=(-1)^{x}, \quad \eta(\hat{z})=(-1)^{y}
$$

are the Dirac $\vec{\alpha}$ matrices for staggered fermions [23]. The gauge field $U[\vec{r}, \hat{n}]$ is associated with the link $[\vec{r}, \hat{n}]$ ant it is a group element in the fundamental representation of $S U\left(N_{C}\right)$. In the strong coupling expansion the electric field Hamiltonian $H_{e}$, (2), is the unperturbed Hamiltonian while the interaction Hamiltonian between quarks and gauge fields $\tilde{H}_{q}$, (3), and the magnetic Hamiltonian $H_{m}$, (44), are treated as perturbations. An important feature of the Hamiltonian (11) is its invariance under translation by a single link which plays the role of a discrete chiral symmetry [8]. It takes even sites into odd sites (even (odd) sites are those with $x+y+z$ even (odd)). The pertinent lattice transformations have the form $\Psi(r) \rightarrow \Psi(r+\hat{x})(-1)^{y}, \Psi(r) \rightarrow \Psi(r+\hat{y})(-1)^{z}$ and $\Psi(r) \rightarrow \Psi(r+\hat{z})(-1)^{x}$. In momentum space the last equation can be written as $q \rightarrow e^{i k_{z}} \gamma_{5} \tau_{3} q$, which in the continuum limit, where $k_{z}$ is infinitesimal, becomes $q \rightarrow \gamma_{5} \tau_{3} q$. The other two transformations yield $q \rightarrow \gamma_{5} \tau_{2} q$, $q \rightarrow \gamma_{5} \tau_{1} q$.

$H_{e}$ has two degenerate ground states corresponding to those of the spin $N_{C} / 2$ antiferromagnetic Ising model. One state may be obtained from the other by interchanging odd and even sites; choosing one of these two vacua leads to the spontaneous breakdown of the 
chiral symmetry. In the thermodynamic limit the two ground states are not mixed to any finite order of perturbation theory [10] and thus, in the perturbative expansion, one only has to consider diagonal matrix elements and, consequently, perturbation theory for nondegenerate states. The ground state energy has been evaluated in the strong coupling regime in [1] up to the fourth order in the strong coupling expansion and for a generic value of $N_{C}$. The order parameter evidencing discrete chiral symmetry breaking is the mass operator $\mathcal{M}=\bar{\psi}(\vec{r}) \psi(\vec{r})$ since it acquires a nonzero expectation value giving rise to the chiral condensate. In the staggered fermion formalism the pertinent lattice operator is given by

$$
\mathcal{M}=-\frac{1}{M a^{3}} \sum_{\vec{r}}(-1)^{x+y+z} \psi_{A}^{\dagger}(\vec{r}) \psi_{A}(\vec{r})
$$

One has to evaluate the expectation value of $\mathcal{M}$ on the perturbed states $\mid p_{0}>$ generated by applying $\tilde{H}_{q}$ to the ground state $\mid 0>$. One has $\left|p_{0}>=\right| 0>+\left|p_{0}^{(1)}>+\right| p_{0}^{(2)}>$, where

$$
\begin{aligned}
\mid p_{0}^{(1)}> & =\frac{\Pi_{0}}{E_{0}^{(0)}-H_{e}} \tilde{H}_{q} \mid 0> \\
\mid p_{0}^{(2)}> & =\frac{\Pi_{0}}{E_{0}^{(0)}-H_{e}} \tilde{H}_{q} \frac{\Pi_{0}}{E_{0}^{(0)}-H_{e}} \tilde{H}_{q}\left|0>+\frac{\Pi_{0}}{E_{0}^{(0)}-H_{e}} H_{m}\right| 0>.
\end{aligned}
$$

The lattice chiral condensate is then given by

$$
\chi_{L}=\frac{<0|\mathcal{M}| 0>+<p_{0}^{(1)}|\mathcal{M}| p_{0}^{(1)}>+<p_{0}^{(2)}|\mathcal{M}| p_{0}^{(2)}>}{<0\left|0>+<p_{0}^{(1)}\right| p_{0}^{(1)}>+<p_{0}^{(2)} \mid p_{0}^{(2)}>}
$$

where

$$
<,>=\left\{\prod_{[\vec{r}, \hat{n}]} \int d U[\vec{r}, \hat{n}]\right\}(,)
$$

is the inner product in the full Hilbert space of the model. $d U$ is the Haar measure on the gauge group manifold and $($,$) the fermion Fock space inner product; \Pi_{0}$ is the projection operator projecting onto states orthogonal to $\mid 0>$.

$\chi_{L}$ may be more conveniently computed by constructing an eigenstate of $H_{e}$ and using it to evaluate the functions $f\left(H_{e}\right)$ appearing in Eq.(9). In order to show the method used to evaluate $\chi_{L}$ [1] we will concentrate on the denominator of Eq.(9). Since the vacuum state $\mid 0>$ is a singlet of the electric field algebra, one has $E^{a}[\vec{r}, \hat{n}] \mid 0>=0$ which, in turn, implies that $H_{e} \mid 0>=0$. Using the left action of the Lie algebra generated by the electric field $E^{a}[\vec{r}, \hat{n}]$ on $U[\vec{r}, \hat{n}], H_{e} \mid 0>=0$ and putting the commutator $\left[H_{e}, U[\vec{r}, \hat{n}]\right] \mid 0>$ in place of $H_{e} U[\vec{r}, \hat{n}] \mid 0>$, one finds

$$
H_{e} U[\vec{r}, \hat{n}]\left|0>=\frac{g^{2}}{2 a} C_{2}\left(N_{C}\right) U[\vec{r}, \hat{n}]\right| 0>,
$$


where $C_{2}=\left(N_{C}^{2}-1\right) / 2 N_{C}$ is the Casimir operator of $S U\left(N_{C}\right) . U[\vec{r}, \hat{n}] \mid 0>$ is then an eigenstate of $H_{e}$ with eigenvalue $g^{2} C_{2}\left(N_{C}\right) / 2 a$. Consequently,

$$
<0\left|\tilde{H}_{q} \frac{1}{\left(E_{0}-H_{e}\right)^{2}} \tilde{H}_{q}\right| 0>=\frac{8 a^{2}}{g^{4} C_{2}^{2}}<0\left|H_{q}^{\dagger} H_{q}\right| 0>.
$$

Taking into account eq.(11) after integration over the link variable $U$ [24] and the fact that $<0 \mid 0>=1$, one finds

$$
<p_{0}^{(1)} \mid p_{0}^{(1)}>=\frac{t^{2}}{g^{4} C_{2}^{2}} N_{C} N
$$

To compute $<p_{0}^{(2)} \mid p_{0}^{(2)}>$ one needs to construct suitable eigenstates of the unperturbed Hamiltonian $H_{e}$ containing two and four link variables $U[\vec{r}, \hat{n}][1]$. Using the eigenvalues of the $f\left(H_{e}\right)$ 's on these eigenstates one finds

$$
\begin{aligned}
<p_{0}^{(2)} \mid p_{0}^{(2)}> & =\frac{4 a^{2}}{g^{4} C_{2}^{2}}\left[\frac{4 a^{2}}{g^{4} C_{2}^{2}}<0\left|H_{q} H_{q}^{\dagger} H_{q} H_{q}^{\dagger}\right| 0>+\frac{2 a^{2}}{g^{4} C_{2}^{2}}\left(<0\left|H_{q} H_{q} H_{q}^{\dagger} H_{q}^{\dagger}\right| 0>\right.\right. \\
& +\frac{2 N_{C}-3}{\left(N_{C}-2\right)^{2}}<0 \mid H_{q} H_{q} \sum_{[\vec{r}, \hat{n}]} \Psi_{A}^{\dagger}(\vec{r}) U_{A B}^{\dagger}[\vec{r}, \hat{n}] \Psi_{B}(\vec{r}+\hat{n}) \\
& \left.\left.\times \Psi_{C}^{\dagger}(\vec{r}) U_{C D}^{\dagger}[\vec{r}, \hat{n}] \Psi_{D}(\vec{r}+\hat{n}) \mid 0>\right)\right] \\
& +\frac{1}{4 g^{8} C_{2}^{2}}<0 \mid \sum_{[\vec{r}, \hat{n}, \hat{m}]} U_{A B}[\vec{r}, \hat{n}] U_{B C}[\vec{r}+\hat{n}, \hat{m}] U_{C D}^{\dagger}[\vec{r}+\hat{m}, \hat{n}] U_{D A}^{\dagger}[\vec{r}, \hat{m}] \\
& \times \sum_{\left[\overrightarrow{r^{\prime}}, \hat{l}, \hat{k}\right]} U_{E F}\left[\overrightarrow{r^{\prime}}, \hat{l}\right] U_{F G}\left[\overrightarrow{r^{\prime}}+\hat{l}, \hat{k}\right] U_{G H}^{\dagger}\left[\overrightarrow{r^{\prime}}+\hat{k}, \hat{l}\right] U_{H E}^{\dagger}\left[\overrightarrow{r^{\prime}}, \hat{k}\right] \mid 0>
\end{aligned}
$$

and the integration over the link variables leads to

$$
\begin{aligned}
<p_{0}^{(2)} \mid p_{0}^{(2)}>= & \frac{t^{4}}{g^{8} C_{2}^{4}}\left[\left(-5 N_{C}-\frac{N_{C}^{2}}{2}\right) N+\frac{N_{C}^{2}}{2} N^{2}+\frac{N_{C}\left(N_{C}-1\right)^{3}}{2\left(N_{C}-2\right)^{2}}\right] \\
& +\frac{N}{g^{8} C_{2}^{2}} .
\end{aligned}
$$

The expectation values of the mass operator $\mathcal{M}$ are computed using a similar procedure and they are given by

$$
\begin{aligned}
& <0|\mathcal{M}| 0>=-\frac{N_{C}}{2 a^{3}} \\
& <p_{0}^{(1)}|\mathcal{M}| p_{0}^{(1)}>=-\frac{t^{2}}{g^{4} C_{2}^{2} a^{3}}\left[-6 N_{C}+\frac{N_{C}^{2}}{2} N\right] \\
& <p_{0}^{(2)}|\mathcal{M}| p_{0}^{(2)}>=-\frac{t^{4}}{g^{8} C_{2}^{4} a^{3}}\left[60 N_{C}+6 N_{C}^{2}-\frac{17}{2} N_{C}^{2} N-\frac{N_{C}^{3}}{4} N\right. \\
& \left.+\frac{N_{C}^{3}}{4} N^{2}+\frac{N_{C}^{3}}{4} N^{2}+\frac{N_{C}\left(N_{C}-1\right)^{3}}{\left(N_{C}-2\right)^{2}}\left(-6+\frac{N_{C}}{4} N\right)\right]-\frac{N_{C}}{2 a^{3}} \frac{N}{g^{8} C_{2}^{2}} .
\end{aligned}
$$


The last contributions in eq.(15) and in eq.(18) come from the magnetic term in $\mid p_{0}^{(2)}>$. Using eqs.(12 18), one finds a non-vanishing chiral condensate for any finite $N_{C}$. For brevity, we do not write the expression of $\chi_{L}$ for a generic value of $N_{C}$, since it may be easily inferred from (9) 18). It is easy to see that, as it should be, all the dependence on the number of lattice links $N$ disappears from the evaluation of $\chi_{L}$ up to the order $t^{4} / g^{8}$ and this is a very non trivial check of our calculations.

Consider now the 't Hooft limit where $g^{2} N_{C}$ is rescaled to $g^{2}$ and $N_{C}$ is then sent to infinity. One gets for the bare lattice chiral condensate

$$
\chi_{L}=-\frac{N_{C}}{a^{3}}\left[\frac{1}{2}-24 \epsilon+864 \epsilon^{2}\right]
$$

where $\epsilon=t^{2} / g^{4}$.

\section{COMPARISON WITH THE RGI CHIRAL CONDENSATE}

To compare the results of the strong coupling expansion with the numerical value of the scale independent RGI chiral condensate determined in [3] one needs to construct a scale independent quantity. This can be done by considering for example the ratio between $\chi_{L}$ which has dimension (mass) ${ }^{3}$ and the mass cube of a meson. In a previous work 1] we derived, in strong coupling, the series expansions for the masses of the low-lying states in the meson spectrum obtaining a good agreement between the experimental values for the meson mass ratios and our lattice results. For the $\rho$ meson we found [1]

$$
m_{\rho}=\frac{g^{2}}{a}\left[\frac{1}{4}+6 \epsilon-203 \epsilon^{2}\right] .
$$

In order to eliminate the dependence on $a^{3}$, one may then consider the ratio between $\chi \equiv$ $-\chi_{L} / N_{C}$ and $m_{\rho}^{3}$

$$
\frac{\chi}{m_{\rho}^{3}}=\frac{1}{g^{6}} \frac{\frac{1}{2}-24 \epsilon+864 \epsilon^{2}}{\left(\frac{1}{4}+6 \epsilon-203 \epsilon^{2}\right)^{3}} .
$$

To compare this strong coupling result with the numerical value of the RGI chiral condensate, the series derived in the strong coupling regime $\left(\epsilon=t^{2} / g^{4} \ll 1\right)$, need to be extrapolated to the region in which $\epsilon \gg 1$, which corresponds to weak coupling. To make this extrapolation possible it is customary to use the Padé approximant method which allows one to extrapolate 
a series beyond its convergence radius. Using $1 / g^{6}=\epsilon^{3 / 2} / t^{3}$, from (21) one gets

$$
\left[\frac{\chi}{m_{\rho}^{3}}\right]^{4 / 3}=\frac{\epsilon^{2}}{t^{4}}\left[\frac{\frac{1}{2}-24 \epsilon+864 \epsilon^{2}}{\left(\frac{1}{4}+6 \epsilon-203 \epsilon^{2}\right)^{3}}\right]^{4 / 3} .
$$

Using the $[0,2]$ Padé approximant, one has

$$
\left[\frac{\chi}{m_{\rho}^{3}}\right]^{4 / 3} \simeq \frac{\epsilon^{2}}{t^{4}} \frac{2^{2 / 3} \times 64}{1+160 \epsilon+7632 \epsilon^{2}} .
$$

For $(\epsilon \rightarrow \infty)$, eq.(23) becomes

$$
\left[\frac{\chi}{m_{\rho}^{3}}\right]^{4 / 3} \stackrel{\epsilon \rightarrow \infty}{\rightarrow}\left(\frac{2^{2 / 3} \times 64}{7632}\right) \frac{1}{t^{4}} .
$$

If one takes for the chiral condensate $\chi$ the RGI numerical value obtained in [3] and for the value of the mass of the $\rho$ meson its experimental value, equating their ratio to (24), one obtains

$$
\left(\frac{2^{2 / 3} \times 64}{7632}\right) \frac{1}{t^{4}}=\left[\frac{\left(\chi_{\mathrm{num}} \pm \Delta \chi_{\mathrm{num}}\right)}{((0.771 \pm 0.0009))^{3}}\right]^{4 / 3},
$$

where $\chi_{\text {num }}$ is the numerical value of the chiral condensate of ref. [3]

$$
\chi_{\text {num }} \pm \Delta \chi=(0.0167 \pm 0.0031) \mathrm{Gev}^{3}
$$

¿From eq.(25) one gets for $t$ the following value

$$
t=1.025 \pm 0.177
$$

The result obtained is close to the expected value of 1, within the error, thus showing very good agreement between our results for the ratio $\chi_{L} / m_{\rho}^{3}$ and the one obtained from the results of [3]. In the r.h.s. of eq.(25) the error on the numerical value of $\chi_{L}$ is due to statistical effects while the error on the mass of $\rho$ is experimental. Of course, for lattice QCD we can only check the consistency of the strong coupling determination of the chiral condensate by comparison of our result with the numerical value of the RGI chiral condensate determined in [3]. A comparison with the "true value" of the continuum chiral condensate is for QCD- not only impossible but also senseless due to the manifest scale and renormalization scheme dependence of the lattice determinations of this quantity [2, 3, 4, 5]. As we shall see immediately after, in the simpler case of the Schwinger model, the strong coupling evaluation of the scale independent ratio between the chiral condensate and the pseudoscalar excitation mass matches very well the exact continuum value. 


\section{CONCLUDING REMARKS}

The reliability of the method used to estimate the accuracy of our computation of the chiral condensate in QCD may be usefully tested in the one flavor Schwinger model for which the value of the chiral condensate and the mass of the pseudoscalar boson in the continuum are known exactly. In fact, one may compute the ratio between the lattice chiral condensate and the lattice pseudoscalar mass and then equate it to its continuum exact counterpart. For the sake of clarity, we briefly recall the results of Berruto et al. 10] for the evaluation of the lattice chiral condensate and the pseudoscalar mass of the strongly coupled one-flavor Schwinger model in the Hamiltonian approach with staggered fermions. In the continuum gauge model the chiral symmetry is broken by the anomaly. In the Hamiltonian lattice formulation of the Schwinger model the axial symmetry is spontaneously broken via a non zero expectation value of the chiral condensate. In the continuum theory it is well known that [25] $<\bar{\psi}(x) \psi(x)>=-e^{\gamma} e_{c} /(2 \pi \sqrt{\pi})$, where $\gamma=0.577 \ldots$ is the Euler constant and $m=e_{c} / \sqrt{\pi}$ is the mass of the pseudoscalar excitation.

A lattice Hamiltonian which, in the continuum limit, reduces to the Schwinger Hamiltonian is

$$
H_{S}=\frac{e_{L}^{2} a}{2} \sum_{x} E_{x}^{2}-\frac{i t}{2 a} \sum_{x}\left(\psi_{x+1}^{\dagger} e^{i A_{x}} \psi_{x}-\psi_{x}^{\dagger} e^{-i A_{x}} \psi_{x+1}\right) \equiv H_{u}+H_{p},
$$

where the fermion fields are defined on the sites, $x=-\frac{N}{2},-\frac{N}{2}+1, \ldots, \frac{N}{2}$, gauge and electric fields, $A_{x}$ and $E_{x}$, on the links $[x, x+1] ; N$ is an even integer. The coefficient $t$ of the hopping term in (28) plays the role of the lattice light speed. In the naive continuum limit, $e_{L}=e_{c}$ and $t=1$.

In the strong coupling limit the electric field Hamiltonian $H_{u}$ is the unperturbed Hamiltonian while the hopping Hamiltonian $H_{p}$ is treated as a perturbation. As in $3+1$ dimensional QCD, there are two degenerate gauge invariant ground states which, in the Coulomb gauge, have the form

$$
\left|\psi>=\prod_{x=\text { even }} \psi_{x}^{\dagger}\right| 0>, \quad\left|\chi>=\prod_{x=\text { odd }} \psi_{x}^{\dagger}\right| 0>
$$

To the fourth order in $\alpha=t / 2 e_{L}^{2} a^{2}$, the perturbative expansion for the ground state energy is given by [10]

$$
E_{\psi}=E_{\psi}^{(0)}+\alpha^{2} E_{\psi}^{(2)}+\alpha^{4} E_{\psi}^{(4)}=\frac{N}{32}-4 N \alpha^{2}+192 N \alpha^{4}
$$


On the lattice the pseudoscalar boson of the continuum Schwinger model is provided by the operator $\mid \theta>=1 /(\sqrt{N}) \sum_{x=1}^{N}\left(\psi_{x}^{\dagger} e^{i A} \psi_{x+1}+\psi_{x+1}^{\dagger} e^{-i A} \psi_{x}\right)$. The energy of this state was computed up to the fourth order in the strong coupling expansion in [10] and its mass is given by subtracting the ground state energy (30) from it

$$
m_{p}=E_{\theta}-E_{\psi}=e_{L}^{2} a\left(\frac{1}{4}+8 \alpha^{2}-576 \alpha^{4}\right) .
$$

The lattice chiral condensate in the staggered fermion formalism may be obtained by considering the mass operator $M(x)=-1 /(N a) \sum_{x=1}^{N}(-1)^{x} \psi_{x}^{\dagger} \psi_{x}$ and evaluating its expectation value on the perturbed state $\mid p_{\psi}>$ generated by applying $H_{h}$ to $|\psi>,| p_{\psi}>=|\psi>+| p_{\psi}^{(1)}>$ $+\mid p_{\psi}^{(2)}>$. A direct computation of the lattice chiral condensate is given in [10] and yields

$$
\chi_{L}=\frac{<p_{\psi}|M| p_{\psi}>}{<p_{\psi} \mid p_{\psi}>}=-\frac{1}{a}\left(\frac{1}{2}-32 \alpha^{2}+1536 \alpha^{4}\right) .
$$

We here compute the ratio between the lattice chiral condensate (32) and the lattice pseudoscalar mass (31) and equate it to its continuum value

$$
-\left(\frac{2}{e_{L}^{2} a^{2}}\right) \frac{\frac{1}{4}-16 z+768 z^{2}}{\frac{1}{4}+8 z-576 z^{2}}=-\frac{e^{\gamma}}{2 \pi},
$$

where $z=\alpha^{2}=\frac{t^{2}}{4 e_{L}^{4} a^{4}}$. Eq.(33) is true only when Padé approximants are used, since the 1.h.s. holds only for $z \ll 1$, while the r.h.s. provides the value of the ratio between the chiral condensate and the pseudoscalar mass obtained when $z \cong \infty$. Using the fourth power of eq.(33) in order to construct the $[0,2]$ Padé approximant for the 1.h.s. of eq.(33), one gets

$$
\left(\frac{\chi_{L}}{m_{p}}\right)^{4}=\frac{256}{t^{4}} \frac{z^{2}}{1+384 z+58368 z^{2}} .
$$

One may now take the limit $z \rightarrow \infty$ obtaining $\left(\chi_{L} / m_{p}\right)^{4}=1 /\left(228 t^{4}\right)$ and, equating it to its continuum counterpart, one gets an equation for $t$

$$
\frac{1}{228 t^{4}}=\left(\frac{e^{\gamma}}{2 \pi}\right)^{4}
$$

¿From eq.(35) this one gets $t=1.03$ for the lattice light velocity; the result lies $3 \%$ above the exact value thus showing that the strong coupling evaluation of the chiral condensate yields a very good result also for this model.

In conclusion, our large $N_{C}$ Hamiltonian approach with staggered fermions evidences that the possible ground state of strongly coupled lattice QCD are those of a spin $N_{C} / 2$ 
antiferromagnetic Ising model; choosing one of the two ground states amounts then to the spontaneous breaking of the discrete chiral symmetry corresponding to translations by a lattice site. As a consequence a non vanishing chiral condensate is found which is the order parameter for the spontaneous breakdown of the chiral symmetry. The bare lattice chiral condensate $\langle\bar{\psi} \psi>$ is computed in the strong coupling region for a general number of colors $N_{C}$ using the method described in 1]. The result obtained is intensive, i.e. independent on the number of lattice links. Then the 't Hooft limit is taken; the ratio between the chiral condensate, obtained in this way, and the third power of the $\rho$ meson mass computed in the 't Hooft limit in [1] is used to provide an estimate of the lattice light velocity, $t$. $t$ turns out to be very close to the expected value of 1 ; this evidences that the large $N_{C}$ limit is a very reliable and relatively simple pathway for the evaluation of the chiral condensate also in the strong coupling region.

[1] G. Grignani, D. Marmottini and P. Sodano, Phys. Rev. D 68, 076003 (2003) arXiv:hep-lat/0306004.

[2] L. Giusti, F. Rapuano, M. Talevi and A. Vladikas, Nucl. Phys. B 538, 249 (1999) arXiv:hep-lat/9807014.

[3] L. Giusti, C. Hoelbling and C. Rebbi, Phys. Rev. D 64, 114508 (2001) [Erratum-ibid. D 65, 079903 (2002)] arXiv:hep-lat/0108007.

[4] P. Hernandez, K. Jansen and L. Lellouch, Phys. Lett. B 469, 198 (1999) arXiv:hep-lat/9907022.

[5] P. Hernandez, K. Jansen, L. Lellouch and H. Wittig, JHEP 0107, 018 (2001) arXiv:hep-lat/0106011.

[6] A. Armoni, M. Shifman and G. Veneziano, arXiv:hep-th/0309013.

[7] L. H. Karsten and J. Smit, Nucl. Phys. B 183, 103 (1981).

[8] T. Banks, S. Raby, L. Susskind, J. B. Kogut, D. R. Jones, P. N. Scharbach and D. K. Sinclair [Cornell-Oxford-Tel Aviv-Yeshiva Collaboration], Phys. Rev. D 15, 1111 (1977).

[9] G. Grignani, D. Marmottini and P. Sodano, Nucl. Phys. Proc. Suppl. 119, 281 (2003) arXiv:hep-lat/0305017.

[10] F. Berruto, G. Grignani, G. W. Semenoff and P. Sodano, Phys. Rev. D 57, 5070 (1998) 
arXiv:hep-lat/9710066.

[11] F. Berruto, G. Grignani and P. Sodano, Phys. Rev. D 62, 054510 (2000) arXiv:hep-lat/9912038.

[12] R. C. Brower and B. Svetitsky, Phys. Rev. D 61, 114511 (2000) [arXiv:hep-lat], F. Berruto, R. C. Brower and B. Svetitsky, Phys. Rev. D 64, 114504 (2001) arXiv:hep-lat/0105016.

[13] J. Smit, Nucl. Phys. B 175, 307 (1980).

[14] J. B. Marston, Phys. Rev. Lett. 61, 1914 (1988);

[15] F. Berruto, G. Grignani, G. W. Semenoff and P. Sodano, Phys. Rev. D 59, 034504 (1999) arXiv:hep-th/9809006.

[16] F. Berruto, G. Grignani, G. W. Semenoff and P. Sodano, Annals Phys. 275, 254 (1999) arXiv:hep-th/9901142.

[17] G. W. Semenoff, Mod. Phys. Lett. A 7, 2811 (1992) arXiv:hep-th/9204047; E. Langmann and G. W. Semenoff, Phys. Lett. B 297, 175 (1992); M. C. Diamantini, P. Sodano, E. Langmann and G. W. Semenoff, Nucl. Phys. B 406, 595 (1993) arXiv:hep-lat/9301014.

[18] G. 't Hooft, Nucl. Phys. B 72, 461 (1974).

[19] G. 't Hooft, Nucl. Phys. B 75, 461 (1974).

[20] M. Teper, arXiv:hep-ph/0203203.

[21] O. Aharony, S. S. Gubser, J. M. Maldacena, H. Ooguri and Y. Oz, Phys. Rept. 323, 183 (2000) arXiv:hep-th/9905111.

[22] M. Kruczenski, D. Mateos, R. C. Myers and D. J. Winters, arXiv:hep-th/0304032

[23] L. Susskind, Phys. Rev. D 16, 3031 (1977).

[24] M. Creutz, "On Invariant Integration Over SU(N)," J. Math. Phys. 19, 2043 (1978).

[25] N. K. Nielsen and B. Schroer, Nucl. Phys. B 120, 62 (1977); K. D. Rothe and J. A. Swieca, Annals Phys. 117, 382 (1979); M. Hortacsu, K. D. Rothe and B. Schroer, Phys. Rev. D 20, 3203 (1979); N. V. Krasnikov, V. A. Matveev, V. A. Rubakov, A. N. Tavkhelidze and V. F. Tokarev, Phys. Lett. B 97 (1980) 103; R. Roskies and F. Schaposnik, Phys. Rev. D 23, $558(1981)$. 